\title{
Ethische Herausforderungen in der forensischen Kinder- und Jugendpsychiatrie. Eine qualitative Beobachtungsstudie und ein Instrument zur Früherkennung und Frühintervention
}

\author{
Jan Schürmann • Mara Mühleck • Christian Perler • Klaus Schmeck • \\ Stella Reiter-Theil
}

Eingegangen: 7. August 2020 / Angenommen: 19. November 2020 / Online publiziert: 27. Januar 2021

(C) Der/die Autor(en) 2021

Zusammenfassung Die forensische Kinder- und Jugendpsychiatrie steht in einem komplexen Spannungsfeld medizinischer, rechtlicher und sozialer Anforderungen. Die ethischen Herausforderungen, die sich daraus für den stationären Maßnahmenvollzug ergeben, sind bisher kaum untersucht, spezifische Hilfestellungen für Behandelnde fehlen. Diese Studie hat zum Ziel, ethische Themenfelder und Probleme in diesem Bereich zu identifizieren und ein Instrument zur Früherkennung und -intervention ethischer Probleme im Klinikalltag zu entwickeln. Methode: Eine systematische Literaturrecherche sowie eine Beobachtungstudie in der Jugendforensik der Universitären Psychiatrischen Kliniken Basel werden durchgeführt. Die Beobachtungsdaten werden mittels qualitativer Inhaltsanalyse ausgewertet. Das Instrument wird auf der Basis der Resultate nach einer neuen Methode entwickelt. In der Literaturrecherche wurden 14 valide Publikationen identifiziert, die hauptsächlich ethische Probleme der Gerichtspsychiatrie thematisieren wie professionelle Rollenkonflikte, ethische Probleme der Diagnostik und Begutachtung oder der Umgang mit vertraulichen Informationen. Empirische oder empirisch-normative Studien fehlen. In der Beobachtungsstudie wurden 24 ethische Themen und spezifische Probleme identifiziert, besonders häufig sind ethische Fragen zu Moralkompetenzen der

Zusatzmaterial online Zusätzliche Informationen sind in der Online-Version dieses Artikels (https:// doi.org/10.1007/s00481-021-00605-6) enthalten.

J. Schürmann, M.A. ( $₫) \cdot$ M. Mühleck, M.A. · Prof. Dr. rer. soc. S. Reiter-Theil

Abteilung Klinische Ethik, Universitätsspital Basel, Universitäre Psychiatrische Kliniken Basel, Universität Basel, Spitalstrasse 22, 4031 Basel, Schweiz

E-Mail: Jan.Schuermann@usb.ch

Dr. med. C. Perler

Klinik für Forensik, Jugendforensik, Universitäre Psychiatrische Kliniken Basel, Basel, Schweiz

Prof. Dr. med. K. Schmeck

Klinik für Kinder und Jugendliche, Abteilung Forschung, Universitäre Psychiatrische Kliniken Basel,

Basel, Schweiz 
Jugendlichen, zur Behandlungsqualität, zum Umgang mit Regeln und Sanktionen und zur Freiheits- und Privatsphäre. Das Instrument zur Früherkennung und -intervention ethischer Probleme (FIEP) enthält die vier Kernelemente Risikofaktoren, Indikatoren, Interventionsplanung und Entscheidungsfindung. Der Maßnahmenvollzug der forensischen Kinder- und Jugendpsychiatrie offenbart ein breites Spektrum ethischer Themen mit spezifischen ethischen Problemen, welche in der Literatur bisher nur unzureichend untersucht sind. Das hier entwickelte Instrument FIEP unterstützt Behandelnde dabei, ethische Probleme frühzeitig zu erkennen und effizient zu bearbeiten.

Schlüsselwörter Forensische Kinder- und Jugendpsychiatrie · Früherkennung • Frühintervention · Präventive Ethik

\title{
Ethical challenges in child and adolescent forensic psychiatry. Observational study and screening instrument
}

\begin{abstract}
Background and aim Child and adolescent forensic psychiatry is fraught with complex medical, legal, and social tensions. The ethical challenges this entails for inhospital treatment have hardly been investigated, and specific support for health care professionals is lacking. This study identifies ethical issues and problems in this area and develops a tool for early detection and intervention of ethical problems in clinical practice.

Methods A systematic literature search and an observational study in adolescent forensics at the University Psychiatric Clinics Basel are conducted. The observation data are evaluated using a qualitative content analysis. The instrument is developed based on the results using a new method.

Results In the literature search, 14 valid publications were identified that mainly address ethical conflicts in legal psychiatry, such as professional role conflicts, ethical problems in diagnostics and assessment, or confidentiality issues. Empirical or empirical-normative studies are missing. In the observational study, 24 ethical issues and specific conflicts were identified. Ethical questions about moral competencies of the adolescents, the quality of treatment, the handling of rules and sanctions, and freedom and privacy are particularly common. The Instrument for Early Detection and Intervention of Ethical Problems (FIEP) contains the four core elements risk factors, indicators, intervention planning and decision-making.

Conclusions Inhospital treatment in forensic child and adolescent psychiatry reveals a broad spectrum of ethical issues with specific ethical problems, which are hardly investigated in literature so far. The FIEP instrument developed here supports health care practitioners in recognizing ethical problems at an early stage and addressing them efficiently.
\end{abstract}

Keywords Child and adolescent forensic psychiatry · Early detection · Early intervention · Preventive ethics 


\section{Hintergrund und Fragestellungen}

Der Umgang mit jugendlichen Straftätern ${ }^{1}$ in der Schweiz stand aufgrund von Einzelfällen wiederholt im Fokus von Öffentlichkeit und Politik. So z. B. im Fall von „Carlos“, einem jugendlichen Intensivtäter, für welchen die Jugendanwaltschaft 2013 eine maßgeschneiderte Maßnahme anordnete (Jositsch et al. 2013). Nachdem die Kosten $^{2}$ für diese Maßnahme publik geworden waren, entstand eine Debatte über den Aufwand der Resozialisation von Intensivtätern. Auf Druck der Öffentlichkeit wurde die bis dahin erfolgreiche Maßnahme abgebrochen und der Jugendliche inhaftiert - rechtswidrig, wie das Bundesgericht später feststellte. Untersuchungen ergaben, dass der Jugendliche teilweise erniedrigenden Haftbedingungen ausgesetzt war. Zuvor wurde der damals 16-Jährige in einer psychiatrischen Einrichtung nach einem Suizidversuch 13 Tage lang mechanisch fixiert und zwangsweise mediziert, was in einem späteren Gutachten als Misshandlung eingestuft wurde. Aufgrund mehrerer Delikte im Strafvollzug, u. a. versuchte schwere Körperverletzung von Beamten, wurde der inzwischen 24-Jährige 2019 zu einer stationär-therapeutischen Maßnahme und einer Freiheitsstrafe von knapp fünf Jahren verurteilt.

Der Fall ist in vielerlei Hinsicht außergewöhnlich, dennoch macht er einige Eigenheiten des schweizerischen Jugendstrafvollzugs deutlich. Erstens verweist der Fall auf den Grundsatz des Schweizer Jugendstrafgesetzes (JStG), Strafen mit erzieherischen oder therapeutischen Maßnahmen zu verbinden, um Entwicklungsdefizite zu beheben, psychische Erkrankungen zu behandeln und die Resozialisation zu fördern. Dazu können Aufsicht, persönliche Betreuung, ambulante oder stationäre Behandlungen angeordnet werden. Der Freiheitsentzug in einem Gefängnis kann dabei zugunsten einer Unterbringung, z. B. in einer jugendforensischen Einrichtung, aufgehoben werden (Manzoni et al. 2018). Zweitens macht der Fall deutlich, wie stark die Wahrnehmung von Jugendkriminalität und bisweilen auch die Strafverfolgung durch die mediale Berichterstattung beeinflusst wird. Forderungen nach höheren Strafen finden in der Schweizer Bevölkerung jeweils breite Zustimmung (Baier 2019b). Regelmäßig kommt es zu politischen Vorstößen zur Verschärfung des Strafrechts. ${ }^{3}$ Die Anzahl der Verurteilungen von Minderjährigen aufgrund von Gewaltstraftaten hat sich zwischen 2010 und 2015 allerdings halbiert $(-51 \%)$, wobei seit 2015 wieder ein leichter Anstieg ( $+14 \%$ bis 2018$)$ zu verzeichnen ist. ${ }^{4}$ Die Rückfallrate verurteilter Jugendlicher im Erwachsenenalter lag 2017 bei $26 \%$ - relativ tief im internationa-

\footnotetext{
${ }^{1}$ In diesem Artikel wird aufgrund der besseren Lesbarkeit das generische Maskulinum verwendet.

2 Die Maßnahme, die u.a. Betreuung, Wohnung, Privatlehrer, Kampfsporttraining und Taschengeld umfasste, kostete rund $25.000 €$ im Monat.

${ }^{3}$ Diese waren bisher erst bei Erwachsenen erfolgreich, so z. B. bei der lebenslänglichen Verwahrung von gefährlichen und nicht therapierbaren Sexual- und Gewaltstraftätern, der systematischen Abschiebung von ausländischen Straftätern oder einem restriktiveren Umgang mit Hafturlauben oder bedingten Entlassungen.

${ }^{4}$ Vgl. Jugendstrafurteilsstatistik des Schweizer Bundesamts für Statistik (https://www.bfs.admin.ch/bfs/ de/home/statistiken/kriminalitaet-strafrecht/erhebungen/jusus.html, zugegriffen: 30. Dez. 2020). Dunkelfeldbefragungen liefern für den Zeitraum 2006-2014 widersprüchliche Ergebnisse, es gibt jedoch Indizien für einen leichten Anstieg der Jugenddelinquenz nach 2014 (Baier 2019a).
} 
len Vergleich. ${ }^{5}$ Drittens zeigt der Fall die Chancen maßgeschneiderter Behandlungen und die Risiken, wenn diese ausbleiben. Jugendliche Straftäter sind in ihrer Biografie besonders häufig psychosozialen Belastungen wie emotionaler Vernachlässigung, häuslicher Gewalt oder Trennung der Eltern ausgesetzt (Aebi 2019). Die Prävalenz psychischer Störungen unter inhaftierten Jugendlichen ist in der Schweiz (wie auch international) sehr hoch (90\%), und das Rückfallrisiko solcher Jugendlicher für Gewaltdelikte ist höher als bei psychisch unauffälligen Jugendlichen (Bessler et al. 2019). Nicht behandelte Entwicklungsdefizite oder psychische Störungen können sich unter Haftbedingungen intensivieren und den Strafvollzug an seine Grenzen bringen, was wiederum ethisch fragwürdige Praktiken wie Erniedrigung, Zwang oder Misshandlung begünstigt.

Die Versorgung von jugendlichen Straftätern mit psychischer Beeinträchtigung zu verbessern, ist ein zentrales Anliegen der forensischen Kinder- und Jugendpsychiatrie (FKJP) (Souverein et al. 2019). Die Universitären Psychiatrischen Kliniken Basel (UPK) eröffneten zu diesem Zweck 2011 den Bereich Jugendforensik, der Abteilung und Ambulanz umfasst. Die Abteilung ist die einzige stationäre Jugendforensik in der Schweiz mit insgesamt zehn Behandlungsplätzen für Jugendliche beider Geschlechter zwischen 10 und 25 Jahren und betreut Jugendliche nach einem multimodalen Versorgungskonzept. ${ }^{6}$ Die Ambulanz ist auf Abklärung, Begutachtung und ambulante Behandlung von straffälligen oder sozial auffälligen Kindern, Jugendlichen und jungen Erwachsenen spezialisiert.

Aus ethischer Perspektive steht die FKJP in einem komplexen Spannungsfeld medizinischer, rechtlicher und sozialer Anforderungen: Als psychiatrische Disziplin ist sie dem individuellen Patienten verpflichtet, u. a. also Patientenwohl und -wille. Minderjährige, psychisch auffällige Straftäter sind besonders vulnerabel, was zusätzliche therapeutische Sorgfalt und Rücksichtnahme erfordert. Zudem besteht eine Verantwortung für Ausbildung und Erziehung, sofern Regelschule und Eltern diese nicht wahrnehmen können. Daneben sind auch die Rechte und Lebensverhältnisse der Angehörigen angemessen zu berücksichtigen. Als forensische Disziplin ist die FKJP zu Zweckdienlichkeit und Wahrheit gegenüber dem Gericht verpflichtet, insbesondere zur Erhebung und Offenlegung von patientenbezogenen Informationen. Als gesellschaftliche Institution steht die FKJP im Dienst von Politik und Gesellschaft, den Vollzug von Freiheitsstrafen sicherzustellen, die Öffentlichkeit vor weiteren Delikten zu schützen und den Täter zur Resozialisierung zu befähigen.

In diesem Artikel soll zunächst deskriptiv untersucht werden, welche ethischen Probleme sich angesichts der normativen Komplexität im Maßnahmenvollzug der

\footnotetext{
5 In Deutschland lag die Rückfallrate nach 3 Jahren für 2010-2013 nach Jugendstrafrecht sanktionierte Personen bei $41 \%$ (Jehle et al. 2016). Bei mehrfach verurteilten und inhaftierten Jugendlichen liegt die Rückfallrate jeweils deutlich höher.

${ }^{6}$ Das Behandlungskonzept umfasst psychiatrische und psychopharmakologische und -therapeutische Therapie, Familientherapie, sozialpädagogische Therapie, schulischen Unterricht, Sport sowie Arbeits-, Ergo-, Physio- und Musiktherapie. Zuweisungen können durch strafrechtliche oder zivilrechtliche Behörden sowie freiwillig durch die Jugendlichen oder deren Familien selbst erfolgen. Jugendliche werden auch zur stationären Begutachtung aufgenommen sowie für Kriseninterventionen aus Gefängnissen oder Institutionen der gesamten Schweiz.
} 
FKJP stellen. ${ }^{7}$ Dazu werden die Ergebnisse einer systematischen Literaturrecherche zu ethischen Problemen in der FKJP und einer Beobachtungsstudie zu ethischen Problemen in der Abteilung Jugendforensik UPK vorgestellt. Aus normativ-ethischer Perspektive stellt sich Behandelnden und Betrachtern die Frage, wie im klinischen Alltag mit diesen Problemen angemessen umzugehen ist. Institutionell besteht eine Antwort in der zunehmenden Etablierung von Strukturen der ethischen Unterstützung an psychiatrischen Kliniken (Ackermann et al. 2016; Wollenburg et al. 2020). ${ }^{8}$ In diesem Artikel wird als Ergänzung zu fallbezogenen Ethikberatungen ein präventiver Ansatz vorgeschlagen. Präventive Ansätze in der klinischen Ethik sind in der US-amerikanischen Literatur unter dem Namen „Preventive Ethics“ bekannt. Ihr Ziel ist, die Qualität in der Patientenversorgung zu verbessern, indem ethische Probleme auf einer systemischen Ebene proaktiv und präventiv bearbeitet werden im Unterschied zu fallbasierten und reaktiven Formen der Ethikberatung. Dazu werden Risikofaktoren, Indikatoren und Auslöser für ethische Probleme ermittelt und Interventionen entwickelt, die solche Probleme frühzeitig aufzudecken, zu adressieren und $\mathrm{zu}$ verhüten helfen (Foglia et al. 2012; Pavlish et al. 2013). Auf der Basis unserer Studienergebnisse wird ein Instrument zur Früherkennung und -intervention von ethischen Problemen (FIEP) vorgestellt. FIEP soll Behandelnden in der Jugendforensik helfen, ethische Probleme frühzeitig zu erkennen und geeignete Interventionen $\mathrm{zu}$ planen. ${ }^{9}$

\section{Methodik}

\section{Systematische Literaturrecherche}

Für die systematische Literaturrecherche wurde in den Datenbanken PubMed, PubPsych, PsycINFO mit PSYNDEXplus, Web of Science und BELIT ein vergleichbarer Suchalgorithmus verwendet. ${ }^{10}$ Als Suchstrategie wurde festgelegt, die von den Autoren festgelegten Schlüsselbegriffe in einer Freitextsuche zu verwenden und die Treffer anschließend anhand der Zusammenfassung zu validieren. Dazu musste eine Zusammenfassung vorliegen, die sich explizit auf den Maßnahmenvollzug in der FKJP und auf moralische oder ethische Themen, Normen, Prinzipien,

\footnotetext{
${ }^{7}$ Im Fokus steht die therapeutische Praxis des Maßnahmenvollzugs (,,correctional psychiatry“), nicht die forensische Begutachtung für das Gericht (,legal psychiatry“).

8 Diese Strukturen unterstützen Behandelnde bei der ethischen Entscheidungsfindung im klinischen Alltag. Die klinische Ethik stellt dazu verschiedene Ansätze bereit; bisher in der Psychiatrie erprobte Ansätze sind Ethikberatung bzw. Ethikkonsultation (Schürmann und Reiter-Theil 2018), Ethikberatung in Ethikkomitees (Syse et al. 2016), ethische Fall-Deliberation (Voskes et al. 2016), Ethikvisiten (Silén et al. 2016) und projektbezogene Ethikunterstützung (Meyer und Reiter-Theil 2016). In den UPK bietet die Abteilung Klinische Ethik verschiede Formen der ethischen Unterstützung an (Reiter-Theil 2016).

9 Die inhaltlich-ethische Einschätzung der identifizierten Problemfelder muss aus Platzgründen an anderer Stelle erfolgen. Eine exemplarische Einschätzung zu häufig beobachteten Problemen ist im elektronischen Zusatzmaterial unter Nr. III zu finden.

10 Suchalgorithmus in PubMed: (,forensic psychiatry“ AND (youth OR adolescent OR juvenile OR child)) AND (ethic* OR moral).
} 
Theorien o. Ä. bezieht. ${ }^{11}$ Die Recherche orientierte sich an den methodischen Grundsätzen des PRISMA-Statements (Moher et al. 2009). Die in den Publikationen genannten Themen wurden von zwei Forschenden gesichtet, paraphrasiert und anschließend nach Prinzipien geordnet, orientiert am Vier-Prinzipien-Ansatz von Beauchamp und Childress (2013). ${ }^{12}$ Für Dokumentation und Auswertung wurden Clarivate EndNote X9, Microsoft Excel 365 und IBM SPSS Statistics 26 verwendet.

\section{Beobachtungsstudie mit qualitativer Inhaltsanalyse}

Für die Beobachtungsstudie wurden im Zeitraum von April bis September 2016 vier verschiedene Gesprächsformate der Jugendforensik besucht: interne und externe Standortbestimmungen ( $n=13$ bzw. $n=2)$, Übergaben $(n=20)$ und Oberarztvisiten $(n=4) .{ }^{13}$ Drei Forschende erstellten in nicht-teilnehmender Beobachtung 39 Textdokumente (Reiter-Theil 2004; Thierbach und Petschick 2014). Der Auftrag lautete, ethisch relevante Kommunikation in einem Beobachtungsprotokoll festzuhalten. Dieses wurde in der Nachbearbeitung ggf. anhand der Falldokumentation und/ oder einer Besprechung im Forschungsteam ergänzt und finalisiert. Zur Analyse der Textdokumente wurde die qualitative Inhaltsanalyse mittels inhaltlicher Strukturierung nach Mayring (2010) verwendet (Mayring 2010, S. 92-99). ${ }^{14}$ Analysiert wurde (1.) der ethische Inhalt des Kommunikationstextes, d.h. ethische Themen oder Probleme, auf welche der Text explizit oder implizit verweist („Inhalt“), (2.) die pragmatische Form des Kommunikationstextes, d.h. wie der ethische Inhalt thematisiert wird (,Kommunikationsform“), und (3.) die Struktur der Handlungssubjekte, auf die der ethische Inhalt explizit oder implizit verweist („Handlungsstruktur“). Für die Analyse wurde MAXQDA 2020 von VERBI verwendet.

Alle Probanden wurden über den Zweck der Studie aufgeklärt und haben freiwillig in die Teilnahme eingewilligt. Alle Daten wurden vollständig anonymisiert und verschlüsselt. Für diese Studie wurden keine Daten erhoben, die unter das Schweizerische Humanforschungsgesetz (HFG, Art. 2) fallen, insbesondere keine gesundheitsspezifischen Personendaten. Eine Prüfung der Studie durch die zuständige Ethikkommission Nordwest- und Zentralschweiz (EKNZ) war daher nicht erforderlich.

\footnotetext{
11 Nicht berücksichtigt wurden folglich Publikationen, die sich ausschließlich auf die forensische Begutachtung für das Gericht beziehen, zur Forschung mit jugendlichen Straftätern oder zum (nicht-psychiatrischen) Jugendstrafvollzug.

12 Die Prinzipien Hilfeleisten (beneficence) und Nichtschaden (nonmaleficence) wurden zusammengenommen, da die Themen für eine eindeutige Zuordnung nicht hinreichend spezifisch beschrieben sind.

13 Teilnehmende bei (a.) internen Standortbestimmungen: Oberarzt, Therapeuten, Pflegefachpersonen, Pädagogen; (b.) externen (zusätzlich): Angehörige, Jugendstaatsanwalt, ggf. gesetzliche Vertretung, Jugendlicher (in zweitem Teil); (c.) Übergaben: Pflegefachpersonen, Nachtwachen; (d.) Oberarztvisiten: Oberarzt, Bezugspflegefachperson, Psychologe, Jugendliche (einzeln in zweitem Teil).

14 Die Kategorienbildung erfolgt einerseits deduktiv, auf der Grundlage einer Pilotstudie zu ethischen Themen in der Jugendpsychiatrie und der Resultate der vorhergehenden Literaturrecherche, andererseits induktiv während der Codierung des Materials.
} 


\section{Entwicklung eines Instruments zur Früherkennung und -intervention von ethischen Problemen}

Instrumente zur Früherkennung und -intervention von ethischen Problemen wurden bisher für die Bereiche der Intensiv- und Notfallmedizin entwickelt (Anderson-Shaw et al. 2007; Morgenstern 2005; Pavlish et al. 2015b; Scheffold et al. 2012). Für die Entwicklung solcher Instrumente gibt es bisher kein standardisiertes Vorgehen. Hier wurde folgendermaßen vorgegangen, um das Instrument auf der Grundlage von vier Kernelementen zu entwickeln: (1.) Ethische Risikofaktoren ${ }^{15}$ : Durchführung einer Literaturrecherche zu ethischen Risikofaktoren in der Patientenversorgung; Priorisierung, Adaption und Ergänzung der Risikofaktoren für die FKJP; Erstellen eines Katalogs der Risikofaktoren; (2.) Ethische Indikatoren ${ }^{16}$ : Durchführung einer systematischen Literaturrecherche und einer Beobachtungsstudie zu ethischen Themen in der FKJP (s. oben); Synthetisieren der ethischen Indikatoren; Erstellen eines Katalogs der Indikatoren; (3.) Interventionsplanung: Adaptierung des Stufenmodells der Leitlinie zu Entscheidungen am Krankenbett METAP für die FKJP (Albisser Schleger et al. 2019); ${ }^{17}$ (4.) Entscheidungsfindung: Entwicklung eines Verfahrens zur ethischen Entscheidungsfindung auf der Basis langjähriger Erfahrung der $\mathrm{Au}-$ toren in der Ethikberatung. Alle Kernelemente wurden von den Autoren und der Leitung der Abteilung Jugendforensik geprüft, konsentiert und anschließend in einem Arbeitsbogen zusammengestellt.

\section{Ergebnisse}

\section{Ergebnisse der Literaturrecherche zu ethischen Themen in der FKJP}

Bei der Suche am 05.08.2020 wurden insgesamt 509 Publikationen gefunden, ohne Duplikate 340. Aufgrund fehlender Informationen wurden 11 Publikationen ausgeschlossen. 315 Publikationen wurden mangels Relevanz ausgeschlossen, 14 Publikationen wurden als valide eingeschätzt. ${ }^{18}$ Unter den validen Publikationen befinden sich 11 narrative Übersichtstudien, entweder über ethische Themen in der FKJP allgemein (McPherson 2012; Morrison 1986; Ratner 2002; Sikorski und Kuo 2004; Soulier 2010; Zerby und Thomas 2006) oder mit Schwerpunkt zur forensischen Be-

\footnotetext{
15 Ethische Risikofaktoren sind Merkmale, welche die Wahrscheinlichkeit erhöhen, dass ein ethisches Problem auftritt.

16 Ethische Indikatoren sind Merkmale, welche anzeigen, dass ein ethisches Problem vorliegt.

17 „METAP“ ist ein Akronym, welches für die Worte Module, Ethik, Therapieentscheidung, Allokation und Prozess steht. Die modular aufgebaute Leitlinie stellt einen Prozess zur Bearbeitung schwieriger Therapiesituationen aus ethischer Sicht zur Verfügung, mit besonderem Fokus auf der Zuteilung von Leistungen am Krankenbett. Diese Leitlinie wurde als Modell für FIEP ausgewählt, weil die Leitlinie evidenzorientiert ist, zusammen mit klinischen Fachpersonen entwickelt worden ist, mit niederschwelligen Interventionen ansetzt und bereits mit positivem Ergebnis evaluiert wurde. Zudem waren die Letztautorin dieses Artikels maßgeblich an Entwicklung und Evaluierung sowie der Erstautor an der Evaluierung der Leitlinie beteiligt.

18 PRISMA-Flussdiagramm siehe elektronisches Zusatzmaterial unter Nr. I.
} 
gutachtung (Barth 2003; O'Shaughnessy und Andrade 2008; Quinn 1995; Sadoff und Baird 2011; Schetky 1992). Dazu kommen eine strukturierte Übersichtsstudie zu ethischen Themen (Kaltiala-Heino und Eronen 2015) sowie zwei theoretisch-normative Abhandlungen zur Nachprüfung von forensischen Gutachten (Austin et al. 2011) und zu professionellen Rollenkonflikten (Strous 2009) in der FKJP.

Am häufigsten genannt wird das Thema Professioneller Rollenkonflikt (in $93 \%$ aller Publikationen), gefolgt von Diagnostik und Begutachtung (79\%), Vertraulichkeit (57\%), Schutz von Interessen (50\%), Aufklärung und Einwilligung, Urteils- und Schuldfähigkeit (je 43\%), Behandlungsqualität, Diskriminierung und Stigmatisierung sowie Professionelles Fehlverhalten (je 36\%). ${ }^{19}$

\section{Ergebnisse der Beobachtungsstudie}

Das erstellte Kategoriensystem umfasst sechs Hauptkategorien zum Inhalt und jeweils fünf zu Kommunikationsform und Handlungsstruktur ethischer Themen in der Abteilung Jugendforensik. Die sechs inhaltlichen Hauptkategorien sind: Autonomie, Hilfeleisten und Nichtschaden, Gerechtigkeit, Moralkompetenz, Professionalität und Diagnostik und Einschätzung. Die 24 Unterkategorien wurden unterschiedlich häufig gescort: Sehr oft (in $\geq 67 \%$ aller Besprechungen mindestens einmal, in absteigender Reihenfolge) wurden ethische Inhalte zu den Kategorien Moralisches Verhalten, Behandlungsqualität und Regeln und Sanktionen thematisiert, oft (34-66\%) zu den Kategorien Schaden und Nutzen, Freiheit- und Privatsphäre, Lebensführung, Therapeutische Beziehung, Moralisches Denken, Angst und Überforderung und Risikoeinschätzung. Gelegentlich (11-33\%) wurden u. a. Inhalte zu den Kategorien Aufklärung und Einwilligung, Entweichung, Zwangsmaßnahmen oder Ressourcenallokation thematisiert, selten (1-10\%) hingegen u.a. Vertraulichkeit, Menschenwürde, Resozialisierung, Urteils- und Schuldfähigkeit oder Professionelle Pflichten. Ethische Probleme werden nur in Einzelfällen explizit als ,ethische“ Probleme benannt ( $\leq 1 \%$ aller Codings). In Tab. 1 sind die inhaltlichen Hauptkategorien und Unterkategorien, deren Häufigkeit sowie entsprechende, am häufigsten thematisierten ethischen Probleme (nach Häufigkeit geordnet) dargestellt. ${ }^{20}$

Die ethischen Inhalte wurden jeweils einer Kategorie zur Kommunikationsform und zur Handlungsstruktur zugeordnet. Bei der Kommunikationsform unterscheidet das Kategoriensystem zwischen Bericht, Bewertung, Diskussion, Abklärung und Anweisung. Am häufigsten wurden ethische Inhalte berichtet (64\% aller Codings), gefolgt von einer Bewertung (18\%), Diskussion (10\%), Anweisung (6\%) und Abklärung $(1 \%) .{ }^{21}$ Am häufigsten diskutiert wurden Inhalte $\mathrm{zu}$ den Kategorien $\mathrm{Be}$ -

\footnotetext{
19 Für eine Übersicht vgl. elektronisches Zusatzmaterial unter Nr. II. Zwölf Publikationen enthalten explizite Empfehlungen zum Umgang mit den identifizierten ethischen Themen und sind insofern normativ-ethisch, zwei sind rein deskriptiv. Von den normativen Publikationen weisen sieben ihre normativen Grundannahmen explizit aus: Vier-Prinzipien-Ansatz (3), professionelle Ethik-Codizes (2), Ansätze der philosophischen Ethik (1), Ethik der Schadensvermeidung (1).

20 S. Fußnote 13.

21 Der Rahmen hat einen Einfluss auf die Form der Kommunikation ethischer Themen. So ist in Oberarztvisiten der Anteil an Diskussion, Abklärung und Anweisung höher (31\% aller Codings) als in externen Fallbesprechungen (26\%), internen Fallbesprechungen (19\%) oder Übergaben (8\%).
} 
Tab. 1 Thematisierte ethische Themen und Probleme in der Jugendforensik

\begin{tabular}{|c|c|c|}
\hline Ethisches Thema & Ethische Probleme (Auswahl; nach Häufigkeit geordnet) & Häufigkeit $^{\mathrm{a}}$ \\
\hline \multicolumn{3}{|l|}{ Autonomie } \\
\hline $\begin{array}{l}\text { Freiheits- und } \\
\text { Privatsphäre }\end{array}$ & $\begin{array}{l}\text { Einschränkung der Freiheitssphäre (z. B. Aufenthaltsort, } \\
\text { Ausgang, Besuch, Schlafzeiten, Essen, Körperschmuck, } \\
\text { Aktivitäten, Medienzugang, Taschengeld, Eigentum) } \\
\text { Einschränkung der Privatsphäre (z. B. Zimmerkontrolle, } \\
\text { soziale Kontrolle, Personenkontrolle, Hygienekontrolle) }\end{array}$ & Oft \\
\hline $\begin{array}{l}\text { Aufklärung und } \\
\text { Einwilligung }\end{array}$ & $\begin{array}{l}\text { Probleme der Aufklärung (z.B. fragliche, verzögerte, } \\
\text { fehlerhafte, fehlende oder schwierige Aufklärung) } \\
\text { Probleme der Einwilligung (z.B. Ablehnen der Behand- } \\
\text { lung, „Deals“) }\end{array}$ & Gelegentlich \\
\hline Zwangsmaßnahmen & $\begin{array}{l}\text { Abwägen von Chancen und Risiken von freiheits- } \\
\text { einschränkenden Maßnahmen (z. B. Isolation, Fixierung) }\end{array}$ & Gelegentlich \\
\hline Vertraulichkeit & $\begin{array}{l}\text { Verletzung der therapeutischen Schweigepflicht } \\
\text { Weitergabe vertraulicher Informationen durch Drittper- } \\
\text { sonen }\end{array}$ & Selten \\
\hline Menschenwürde & Gefühl der Demütigung oder Tribunalisierung & Selten \\
\hline \multicolumn{3}{|c|}{ Hilfeleistung und Nichtschaden } \\
\hline Behandlungsqualität & $\begin{array}{l}\text { Abwägen von Chancen und Risken einer Behandlung } \\
\text { oder therapeutischen Maßnahme (z. B. Nebenwirkungen, } \\
\text { Wirkungslosigkeit, Überforderung, Sicherheitsbedenken) } \\
\text { Probleme beim Festlegen von Behandlungszielen (z. B. } \\
\text { fehlende Zustimmung od. Adhärenz, Deals, externe } \\
\text { Auflagen, Uneinigkeit im Team) } \\
\text { Gefährdung des Behandlungserfolgs aufgrund von Drit- } \\
\text { ten (z.B. Mitpatienten, Peergroup, Familie) oder be- } \\
\text { sonderen Umständen (z. B. kognitive Einschränkung, } \\
\text { Verlegung) } \\
\text { Problematischer Patientenwunsch nach medikamentöser } \\
\text { Behandlung (z. B. Sedativa aufgrund von Stress oder } \\
\text { Schlafproblemen) } \\
\text { Ausbleibender Behandlungserfolg/Behandlungsziele auf } \\
\text { der Abteilung nicht zu erreichen (z. B. therapieresistente } \\
\text { Erkrankung, fehlende Therapiebereitschaft) }\end{array}$ & Sehr oft \\
\hline $\begin{array}{l}\text { Schaden und Nut- } \\
\text { zen }\end{array}$ & $\begin{array}{l}\text { Drohungen oder Gewalt von Jugendlichen gegen Mitpa- } \\
\text { tienten oder Behandelnde } \\
\text { Einschätzen von Chancen und Risiken des Kontakts zu } \\
\text { Familienangehörigen } \\
\text { Einschätzen von Chancen und Risiken der freien Persön- } \\
\text { lichkeitsentfaltung (z. B. gewaltverherrlichende Parolen, } \\
\text { destabilisierendes soziales Umfeld) } \\
\text { Sexuelle Belästigung von Patienten durch Mitpatienten } \\
\text { Selbstgefährdung (z.B. Selbstverletzung, Suizidversuch) }\end{array}$ & Oft \\
\hline Lebensführung & $\begin{array}{l}\text { Jugendlicher formuliert schwer realisierbare oder frag- } \\
\text { würdige Berufs- oder Lebensziele (z. B. Berufsziel Infor- } \\
\text { matiker, Beitritt zu fundamentalistischen Gruppierungen) } \\
\text { Jugendlicher will sich nicht auf therapeutisches Setting } \\
\text { einlassen und strebt einen Institutionswechsel an (z. B. } \\
\text { Gefängnis, offener Vollzug) } \\
\text { Probleme bei der Planung einer Anschlusslösung (z.B. } \\
\text { fehlende Institutionen oder Wohnmöglichkeiten, fehlen- } \\
\text { de Arbeitsmöglichkeiten, Zeitdruck, Finanzierung) } \\
\text { Jugendlicher fühlt sich auf der Abteilung zu Hause (Hos- } \\
\text { pitalismus) }\end{array}$ & Oft \\
\hline
\end{tabular}


Tab. 1 (Fortsetzung)

\begin{tabular}{|c|c|c|}
\hline Ethisches Thema & Ethische Probleme (Auswahl; nach Häufigkeit geordnet) & Häufigkeit $^{\mathrm{a}}$ \\
\hline $\begin{array}{l}\text { Angst und Überfor- } \\
\text { derung }\end{array}$ & $\begin{array}{l}\text { Angst des Jugendlichen (z. B. vor Sanktionen, davor } \\
\text { Fehler zu machen, vor Stigmatisierung, vor Beziehungs- } \\
\text { verlust, vor Krisen, davor nach dem Austritt zu versagen) } \\
\text { Überforderung des Jugendlichen (z. B. in der Schule, im } \\
\text { Kontakt mit der Familie) } \\
\text { Sicherheitsbedürfnis der Behandelnden gegenüber Pati- } \\
\text { enten }\end{array}$ & Oft \\
\hline Grundbedürfnisse & $\begin{array}{l}\text { Nichterfüllen des Grundbedürfnisses nach Sexualität } \\
\text { oder körperlicher Intimität (z. B. Verbot sexueller Kon- } \\
\text { takte zwischen Patienten auf der Abteilung) }\end{array}$ & Selten \\
\hline \multicolumn{3}{|l|}{ Gerechtigkeit } \\
\hline $\begin{array}{l}\text { Regeln und Sank- } \\
\text { tionen }\end{array}$ & $\begin{array}{l}\text { Abwägen und Festlegen von Sanktionen nach Regel- } \\
\text { verstoß (z. B. bei übermäßigem Zigarettenkonsum, bei } \\
\text { wirkungslosen Sanktionen, bei Gefahr einer Eskalation) } \\
\text { Fragliche Angemessenheit von Sanktionen (z.B. Kollek- } \\
\text { tivstrafe, Strafe fürs Diskutieren) } \\
\text { Zweckentfremdung von Sanktionen (z. B. Isolation zum } \\
\text { Aggressionsabbau, Zimmerrückzug zur Konfliktvermei- } \\
\text { dung) }\end{array}$ & Sehr oft \\
\hline Entweichung & $\begin{array}{l}\text { Entweichung mit Einleitung der Fahndung und geplante } \\
\text { Rückführung } \\
\text { Hinweise auf Entweichung werden nicht erkannt oder } \\
\text { falsch interpretiert } \\
\text { Einschätzen und Vorbereiten auf Fluchtgefahr }\end{array}$ & Gelegentlich \\
\hline Ressourcenallokation & $\begin{array}{l}\text { Medikament aufgrund der Kosten auf der Abteilung } \\
\text { nicht verfügbar } \\
\text { Personalknappheit } \\
\text { Fehlen von institutionellen Alternativen zur Abteilung }\end{array}$ & Gelegentlich \\
\hline $\begin{array}{l}\text { Diskriminierung } \\
\text { und Stigmatisie- } \\
\text { rung }\end{array}$ & $\begin{array}{l}\text { Diskriminierendes Verhalten eines Jugendlichen (z. B. } \\
\text { Rassismus, Sexismus) } \\
\text { Jugendliche als Opfer und/oder Täter von Mobbing }\end{array}$ & Selten \\
\hline Resozialisierung & $\begin{array}{l}\text { Einschätzen von Risiko und Konsequenzen einer Aus- } \\
\text { schaffung aufgrund eines Delikts } \\
\text { Einschätzen der Wahrscheinlichkeit einer gelingenden } \\
\text { Resozialisation }\end{array}$ & Selten \\
\hline \multicolumn{3}{|l|}{ Moralkompetenz } \\
\hline $\begin{array}{l}\text { Moralisches Ver- } \\
\text { halten }\end{array}$ & $\begin{array}{l}\text { Einschätzen von Fähigkeiten des moralischen Verhaltens } \\
\text { (z.B. Aggressionskontrolle, Bindungsfähigkeit, Koope- } \\
\text { rationsbereitschaft, Selbstbeherrschung, Authentizität, } \\
\text { Selbstfürsorge, Freundlichkeit, Aufrichtigkeit) }\end{array}$ & Sehr oft \\
\hline $\begin{array}{l}\text { Moralisches Den- } \\
\text { ken }\end{array}$ & $\begin{array}{l}\text { Einschätzen von Fähigkeiten des moralischen Denkens } \\
\text { (z.B. Urteilskraft, Tateinsicht, Reuefähigkeit, Empathie- } \\
\text { vermögen) }\end{array}$ & Oft \\
\hline Moralische Gefühle & $\begin{array}{l}\text { Einschätzen von Fähigkeiten des moralischen Fühlens } \\
\text { (z.B. Scham, Gefühlskälte) }\end{array}$ & Gelegentlich \\
\hline
\end{tabular}


Tab. 1 (Fortsetzung)

\begin{tabular}{|c|c|c|}
\hline Ethisches Thema & Ethische Probleme (Auswahl; nach Häufigkeit geordnet) & Häufigkeit $^{\mathrm{a}}$ \\
\hline \multicolumn{3}{|l|}{ Professionalität } \\
\hline $\begin{array}{l}\text { Therapeutische } \\
\text { Beziehung }\end{array}$ & $\begin{array}{l}\text { Probleme der therapeutischen Beziehung seitens des } \\
\text { Patienten (z. B. Motivationslosigkeit, Verschlossenheit, } \\
\text { Manipulation, Erotisierung, Somatisierung, Vorbehalte) } \\
\text { Probleme der therapeutischen Beziehung seitens der } \\
\text { Behandelnden (z. B. Therapeutenwechsel, Institutions- } \\
\text { wechsel, emotionale Belastung nach Aggression) } \\
\text { Anzeige eines Behandelnden gegen einen Patienten } \\
\text { aufgrund einer Gewalttat }\end{array}$ & Oft \\
\hline $\begin{array}{l}\text { Professionelle } \\
\text { Pflichten }\end{array}$ & $\begin{array}{l}\text { Unklare Fürsorgepflicht der Therapeuten für Patienten } \\
\text { außerhalb der Abteilung } \\
\text { Fragwürdige Konfrontation von Patienten durch Behan- } \\
\text { delnde (z. B. „Fertigmachen“, Beleidigen, Unterstellen) }\end{array}$ & Selten \\
\hline $\begin{array}{l}\text { Professioneller } \\
\text { Rollenkonflikt }\end{array}$ & $\begin{array}{l}\text { Therapeutische vs. pädagogische Aufgaben der Behan- } \\
\text { delnden }\end{array}$ & Selten \\
\hline \multicolumn{3}{|c|}{ Diagnostik und Einschätzung } \\
\hline Risikoeinschätzung & $\begin{array}{l}\text { Risikoeinschätzung bei ethisch relevanten Sachverhalten } \\
\text { (z.B. Selbst- und Fremdgefährdung, Entweichungsrisiko, } \\
\text { Drogenkonsum, Delikte) } \\
\text { Probleme bei der Risikoeinschätzung (z. B. prognosti- } \\
\text { sche Unsicherheit, unsichere Beurteilungsgrundlage, } \\
\text { Meinungsverschiedenheit) }\end{array}$ & Oft \\
\hline $\begin{array}{l}\text { Diagnostik und } \\
\text { Begutachtung }\end{array}$ & $\begin{array}{l}\text { Probleme bei der Diagnostik (Unklarheit/Zweifel bei } \\
\text { der Diagnose, Meinungsverschiedenheit, Unklarheit der } \\
\text { Anamnese) }\end{array}$ & Gelegentlich \\
\hline $\begin{array}{l}\text { Urteils- und } \\
\text { Schuldfähigkeit }\end{array}$ & $\begin{array}{l}\text { Einschätzung der Schuldfähigkeit bei kognitiver Beein- } \\
\text { trächtigung }\end{array}$ & Selten \\
\hline
\end{tabular}

aSkala: Selten in 1-10\% aller Besprechungen mindestens einmal beobachtet, Gelegentlich 11-33\%, Oft $34-66 \%$, Sehr oft $\geq 67 \%$

handlungsqualität (24\% aller Codings in der Kategorie Diskussion), Diagnostik und Begutachtung, Moralisches Verhalten (je 12\%), Regeln und Sanktionen und Risikoeinschätzung (je 9\%). Der Großteil ethisch relevanter Probleme findet sich in Berichten und Bewertungen, die nicht zu einer Diskussion, Abklärung oder Anweisung führen ( $82 \%$ aller Codings). Nur in einem Zehntel aller Fälle werden ethische Probleme diskutiert, noch seltener werden sie Gegenstand weiterer Abklärung oder von Handlungsanweisungen. Bei der Handlungsstruktur wird unterschieden zwischen den Kategorien Individuum, Patient-Bezugsperson, Bezugsumfeld, Institution und Gesellschaft. Am häufigsten bezogen sich ethische Inhalte auf die Beziehung zwischen Patienten und Bezugspersonen (59\% aller Codings), gefolgt von einer Bezugnahme auf das Individuum (30\%), die Institution (6\%), das Bezugsumfeld (5\%) und die Gesellschaft (1\%).

Im Folgenden werden Ergebnisse zu den vier am häufigsten gescorten Unterkategorien vorgestellt. 


\section{Freiheits- und Privatsphäre}

Die Kategorie Freiheits- und Privatsphäre stellt gut zwei Drittel der Befunde in der Hauptkategorie Autonomie. Sie umfasst Aussagen, die sich auf den Schutz bzw. das Aufheben des persönlichen Freiheitsspielraums oder der Privatsphäre der Jugendlichen beziehen. Diese sind insofern ethisch relevant, als den Jugendlichen der Schutz ihrer Freiheits- und Privatsphäre in Form eines Grund- und Menschenrechts zusteht, auch wenn dieses aufgrund ihrer Straffälligkeit und Behandlungsbedürftigkeit eingeschränkt ist. Eher selten geht es dabei um die Frage, ob diese Einschränkungen, d.h. die behördlichen Maßnahmen, grundsätzlich angemessen sind, sondern wie innerhalb des rechtlich-institutionellen Rahmens, der bereits eine Freiheitseinschränkung mit sich bringt, Freiheits- und Privatsphäre bestmöglich gewahrt werden können. Aus ethischer Sicht steht dabei das Freiheitsrecht des Jugendlichen anderen moralischen Verpflichtungen entgegen: Wünsche nach größerer Bewegungsfreiheit kollidieren mit Sicherheitsbedenken; persönliche Aktivitäten können das Freiheitsrecht der Mitpatienten beeinträchtigen, das Zusammenleben gefährden, die Gesundheit schädigen oder gegen Abteilungsregeln verstoßen; die Privatsphäre kann aufgrund von Sicherheitsbedenken oder aus Fürsorge eingeschränkt werden. Zwei Drittel der Befunde zur Freiheits- und Privatsphäre gehen auf Übergaben bei Schichtwechsel zurück. In der Hälfte der Fälle werden diese Inhalte bloß berichtet, nur selten diskutiert. In der Oberarztvisite werden Zimmerstatus und Ausgang für jeden Jugendlichen in Form eines Stufensystems festgelegt - entsprechend gehen fast alle Handlungsanweisungen auf diese Besprechungsform zurück.

\section{Behandlungsqualität}

Qualität und Erfolg einer geplanten oder bereits durchgeführten Behandlung ${ }^{22}$ wird in über zwei Dritteln aller Besprechungen thematisiert. Ethisch relevant ist dabei insbesondere die zugrundeliegende Behandlungspflicht und der Schutz vor unnötigen Risiken oder Überbehandlung. Diese Pflichten beschränken sich nicht auf medizinische und pflegerische Interventionen, sondern betreffen auch pädagogische oder Therapieangebote. Ethisch problematisch können weiterhin Behandlungswünsche sein, die keine Erfolgsaussichten und/oder hohe Risiken haben. Auch Behandlungen oder Therapien, die nicht innerhalb der Institution durchführbar sind, können aufgrund von Sicherheitsbedenken zu ethischen Konflikten führen. Auf institutioneller Ebene kann sich ein weiteres Spannungsfeld zwischen den Behandlungszielen der Jugendforensik und den Forderungen anderer Institutionen (z. B. Jugendanwaltschaft, Betreuer, Eltern) ergeben. Die Intensität, mit welcher die Behandlungsqualität thematisiert wird, ist hoch: Von allen ethischen Inhalten wird die Behandlungsqualität am zweithäufigsten in einer Bewertung, am häufigsten in einer Diskussion angesprochen und ist am häufigsten Gegenstand einer Handlungsanweisung. Drei Viertel dieser Inhalte werden in internen Standortbestimmungen oder Oberarztvisiten thematisiert.

\footnotetext{
${ }^{22}$ Hier in einem weiten Sinn als medizinische, therapeutische, pflegerische oder pädagogische Intervention der Behandelnden verstanden.
} 


\section{Regeln und Sanktionen}

Die Kategorie Regeln und Sanktionen stellt knapp zwei Drittel der Treffer in der Hauptkategorie Gerechtigkeit. Sie umfasst Aussagen, die auf ethisch relevante Handlungsregeln oder Sanktionen verweisen, d.h. die moralische Verpflichtungen zum Ausdruck bringen. In dieser Studie sind das oft Abteilungsregeln, welche das Zusammenleben oder die persönlichen Freiheiten ordnen. Auch Handlungen, die diese Regeln beachten oder gegen diese verstoßen, sind ethisch relevant, insofern sie den zugrundeliegenden moralischen Verpflichtungen nachkommen oder nicht, ebenso das Auferlegen oder Aufheben von Sanktionen. Regeln und Sanktionen können auch unabhängig von ihrem Gehalt ethisch problematisch werden, wenn sie unspezifisch, unbegründet, unverhältnismäßig oder wirkungslos sind - sie widersprechen dann dem Prinzip der Gerechtigkeit. In dieser Hinsicht werden am häufigsten Verhältnismäßigkeit, Wirksamkeit und Spezifizität ${ }^{23}$ von Regeln oder Sanktionen thematisiert. Drei Viertel der Befunde zu Regeln und Sanktionen gehen auf Übergaben zurück. Drei Viertel der Inhalte werden bloß berichtet, nur selten bewertet oder diskutiert.

\section{Moralisches Verhalten}

Über ein Viertel aller ethisch relevanten Kommunikation fällt unter die Kategorie Moralisches Verhalten. Sie umfasst Aussagen, die sich auf ein Verhalten beziehen, das den Entwicklungsstand einer bestimmten Moralkompetenz anzeigt. ${ }^{24}$ Fast immer wird ein Entwicklungsdefizit hinsichtlich eines - meist impliziten - Standards der moralischen Entwicklung konstatiert. Ethisch relevant sind mangelnde Moralkompetenzen, weil sie für gewöhnlich moralisch fragwürdiges Verhalten zur Folge haben. Entsprechend stellen sich auch grundsätzliche moralpädagogische Fragen: Welche Verantwortung haben Behandelnde bezüglich der Moralentwicklung der Jugendlichen? Welche Standards von Moralerziehung und -bildung sollen zugrunde gelegt werden? Wie steht es um die ethische Angemessenheit der Moralerziehung selbst, etwa hinsichtlich prozeduraler Kriterien wie Transparenz, Konsistenz, Fairness oder Verhältnismäßigkeit? Über moralisches Verhalten wird in zwei Dritteln der Fälle bloß berichtet, ein Viertel wird zusätzlich bewertet - vergleichsweise selten wird in den beobachteten Formaten darüber diskutiert. ${ }^{25}$ Abklärungen oder Anweisungen kommen hier nicht vor.

\footnotetext{
${ }^{23}$ Mit Spezifizität ist hier gemeint, ob Regeln oder Sanktionen in spezifischen Situationen eindeutig und sinnvoll sind.

24 Unter Moralkompetenz verstehen die Autoren die Fähigkeit, für gewöhnlich in Übereinstimmung mit bestimmten Vorstellungen des moralisch richtigen Handelns und guten Lebens zu handeln, zu urteilen und zu fühlen.

25 Viele Tugend- oder Lasterbegriffe haben neben ihrem deskriptiven auch einen normativen Gehalt (z.B. Grausamkeit). Als Bewertung werden hier nur solche Aussagen verstanden, die ein zusätzliches normatives Urteil enthalten (z. B. ,Seiner Grausamkeit sollte Einhalt geboten werden.“).
} 


\section{Das Instrument zur Früherkennung und -intervention von ethischen Problemen (FIEP)}

Ethische Risikofaktoren in der Patientenversorgung werden in acht Publikationen untersucht (Anderson-Shaw et al. 2007; Epstein 2012; Heyl 2008; Morgenstern 2005; Pavlish et al. 2015a, 2011, 2015b; Robinson et al. 2017). Insgesamt werden 67 Risikofaktoren identifiziert, von welchen 41 auch für die FKJP relevant sind. Diese können vier Kategorien zugeordnet werden: patientenbezogene Faktoren, teambezogene Faktoren, angehörigenbezogene Faktoren und systemische Faktoren. Pavlish et al. (2015b) haben zudem die Prävalenz der Faktoren im klinischen Alltag untersucht. Für das hier entwickelte Instrument FIEP wurden die vier häufigsten, für die FKJP relevanten Faktoren in jeder Kategorie ausgewählt und für den Kontext der FKJP angepasst. Zudem wurde jeweils ein für die FKJP spezifischer Risikofaktor ergänzt. Auf der Basis der Studienergebnisse wurden spezifische Indikatoren für ethische Probleme in der FKJP ermittelt. $\mathrm{Zu}$ jeder Inhaltskategorie wurde ein qualitativer Indikator formuliert.

Das Stufenmodell zur ethischen Problemlösung der Leitlinie METAP enthält vier Interventionsstufen: eigene Orientierung, Beratung mit (geschultem) Steuergruppenmitglied, ethische Fallbesprechung im Behandlungsteam und professionelle Ethikberatung (Albisser Schleger et al. 2019). Für FIEP wurden Stufe 1 und 4 übernommen; Stufe 2 und 3 wurden durch eine Beratung im Kernteam bzw. im erweiterten Kernteam ersetzt. ${ }^{26}$ Mit PIHAL wurde ein Verfahren entwickelt, welches Behandelnde bei der Bewältigung ethisch komplexer Entscheidungssituationen unterstützt. „PIHAL“ steht als Akronym für die fünf Schritte des Verfahrens: Problem identifizieren, Informationen zusammentragen, Handlungsoptionen festlegen, Angemessenheit prüfen, Lösung finden. ${ }^{27}$

\section{Diskussion}

Ethische Themen im Maßnahmenvollzug der FKJP sind bisher kaum untersucht. Empirische oder Studien, die empirische und normative Methoden verbinden, fehlen gänzlich. Die bestehenden Publikationen nennen ethische Themen nur im Überblick oder sekundär zu ethischen Aspekten der forensischen Begutachtung. Jene Aspekte sind gemäß unserer Studie für die Praxis des Maßnahmenvollzugs nur am Rande relevant. Umgekehrt sind die in der Praxis beobachteten Themen in der Literatur nur unzureichend abgebildet. ${ }^{28}$ Diese Diskrepanz ist einerseits darauf zurückzuführen, dass in der US-amerikanischen Literatur unter ,forensic psychiatry“ hauptsächlich

\footnotetext{
26 Auf der Abteilung Jugendforensik und in den UPK allgemein gibt es keine METAP-Steuergruppe. Dem Kernteam gehören Bezugspflegeperson, Psychotherapeut und Jugendlicher an; das erweiterte Kernteam ist zusätzlich mit pflegerischer und ärztlicher Leitung.

27 Für den Arbeitsbogen zu FIEP s. elektronisches Zusatzmaterial unter Nr. IV.

28 Von den vier am häufigsten beobachteten Themen werden Freiheits- und Privatsphäre sowie Regeln und Sanktionen nur von Kaltiala-Heino und Eronen (2015), Moralkompetenz überhaupt nicht erwähnt, ebenso wenig wie Lebensführung, Angst und Überforderung und Entweichung.
} 
Gerichtspsychiatrie, ,legal psychiatry“, verstanden wird (Niveau und Welle 2018). Andererseits ist sie ein Hinweis auf das frühe Stadium der Ethik-Forschung im Bereich des forensischen Maßnahmenvollzugs - hier sind mehr deskriptiv- und normativ-ethische Untersuchungen nötig.

Diese Beobachtungsstudie zeigt ein breites Spektrum ethischer Themen mit spezifischen Problemstellungen. Fasst man diese in einer disziplinären Ordnung zusammen, so sind dies:

1. Kinder- und Jugendpsychiatrie-ethische Fragen, z. B. Fragen im Zusammenhang mit Moralkompetenz und -erziehung, Fragen zum angemessenen Umgang mit Regeln und Sanktionen, zur Freiheits und Privatsphäre oder zur Lebensplanung der Jugendlichen. ${ }^{29}$

2. Psychiatrie-ethische Fragen, z.B. Fragen zur Angemessenheit therapeutischer Interventionen oder von Zwangsmaßnahmen, Fragen zur therapeutischen Beziehung, zur Qualität von Diagnosestellung, Aufklärung oder Einwilligungseinholung, zur Wahrung der Vertraulichkeit, zur angemessenen Ressourcenallokation oder zur Urteils- oder Schuldfähigkeit der jugendlichen Patienten.

3. Forensisch-ethische Fragen, z. B. Fragen zur Angemessenheit der Risikoeinschätzung, zum Umgang mit Entweichungen, zu den Chancen auf Resozialisierung oder zum Konflikt zwischen forensischen und therapeutischen Pflichten.

Das Auftreten von ethischen Fragen in der FKJP halten wir für unausweichlich und nicht grundsätzlich für ethisch problematisch. Ethische Probleme können jedoch gravierende Folgen für Patienten oder Behandelnde haben, wenn man sie nicht angeht (Pavlish et al. 2013). Nicht alle Probleme erfordern die Bearbeitung in einer Ethikberatung, ethische Probleme sollten so niederschwellig wie möglich gelöst werden. Dies erfordert jedoch ethisch kompetente Mitarbeitende, eine ethisch reflektierte Praxis und geeignete Prozesse zur ethischen Entscheidungsfindung. Ethische Probleme sollen frühzeitig identifiziert, artikuliert, kommuniziert und bearbeitet werden. Unsere Resultate legen nahe, dass dies in den beobachteten Gesprächen nur teilweise gelingt. Hier können niederschwellige und präventive Formen der ethischen Unterstützung ansetzen. Aus organisationsethischer Sicht ergibt sich für uns die Empfehlung, für die ethische Reflexion des Fachpersonal ausreichend Zeit, Raum und Gelegenheit zu schaffen. Selbst in der als Modellstation teilweise privilegierten Abteilung waren die organisatorischen Ressourcen dafür eher knapp und jedenfalls ausbaufähig.

Das hier entwickelte Instrument FIEP stellt eine praxisnahe Möglichkeit der ethischen Unterstützung dar. Im Bereich der klinischen Ethik ist es das erste, welches die vier Kernelemente von Früherkennung und -intervention - Risikofaktoren, In-

\footnotetext{
29 Dieses Themenfeld, das einerseits sehr konkrete Fragen nach dem alltäglichen, geordneten Zusammenleben, andererseits sehr allgemeine Fragen nach dem guten und sinnerfüllten Leben umfasst, findet in der medizinethischen Literatur bisher kaum Erwähnung. Eine normative Grundlage finden diese Themen in den Grundsätzen der UN-Konvention zu den Rechten des Kindes (UNICEF 1989), z. B. im Recht auf Meinungs- und Gedankenfreiheit (Art. 13/14), Recht auf Schutz der Privatsphäre (Art. 16/40), Recht auf Bildung, inkl. moralischer Bildung und der Vorbereitung auf ein verantwortungsbewusstes Leben (Art. 28/29), Schutz vor erniedrigenden Strafen (Art. 37), Recht auf Behandlung mit Achtung vor der Menschenwürde und unter Berücksichtigung altersentsprechender Bedürfnisse bei Freiheitsentzug (Art. 37/40).
} 
dikatoren, Interventionsplanung und Entscheidungsfindung - zusammenführt. Die Indikatoren beruhen auf den Ergebnissen der Beobachtungstudie und sind daher für die Jugendforensik hoch spezifisch. Die Interventionsplanung beginnt bei niederschwelligen, praxisnahen Formen der ethischen Reflexion. Es enthält keine inhaltlich-ethischen Handlungsempfehlungen, im Gegensatz zu ethischen Richtlinien oder professionellen Ethik-Codizes, sondern empfiehlt einen Prozess: ein formales Verfahren zur ethischen Entscheidungsfindung, das der Nutzer selbständig anwenden kann. Führt dies nicht zu einer angemessenen Lösung, ist die nächste Interventionsstufe vorzubereiten. Eine nachhaltige Implementierung des Instruments erfordert neben einer geeigneten Schulung der Mitarbeitenden die Akzeptanz und Präsenz des Instruments, die Unterstützung durch die Leitung, ein (wachsendes) ethisches Klima, ein Bewusstsein für den Bedarf an ethischer Unterstützung und engagierte Mitarbeitende (Meyer-Zehnder et al. 2017).

Eine Limitation dieser Studie liegt darin, dass Gespräche und nicht die eigentliche Maßnahmenvollzugspraxis beobachtet wurden. Zur Beobachtung wurden allerdings relevante Gesprächsformate gewählt, in welchen wesentliche Inhalte der Behandlungspraxis und insbesondere auch ethische Themen diskutiert werden. Diese Gespräche sind in die Vollzugspraxis eingebunden und damit integrativer Teil dieser Praxis. Bei einer direkten Beobachtung der Jugendlichen hätte mit erheblichen Verzerrungseffekten gerechnet werden müssen. Zur Validierung von FIEP läuft 2020-2021 eine Pilotstudie.

\section{Schlussfolgerungen}

Der Maßnahmenvollzug der FKJP steht in einem komplexen Spannungsfeld normativer Anforderungen, was sich in einem breiten Spektrum ethischer Themen und spezifischen ethischen Problemen niederschlägt. In dieser Studie zeigen sich am häufigsten Fragen der Moralkompetenz und -erziehung, zur Behandlungsqualität, zum Umgang mit Regeln und Sanktionen und zur Freiheits- und Privatsphäre. Im Zusammenhang der FKJP wurden diese Themen bisher kaum untersucht. Zum kompetenten Umgang mit ethischen Herausforderungen wird ein präventives und prozedurales Modell vorgeschlagen. Das hier entwickelte Instrument zur Früherkennung und -intervention ethischer Probleme (FIEP) unterstützt Behandelnde dabei, ethische Probleme frühzeitig zu erkennen und effizient zu bearbeiten.

Danksagung Die Autoren danken der Leitung und dem Team der Jugendforensik UPK Basel für die gute Kooperation im Rahmen dieser Studie. Dem Forschungsförderungsfond UPK danken die Autoren für die finanzielle Unterstützung des Projekts.

Funding Open access funding provided by University of Basel

Open Access Dieser Artikel wird unter der Creative Commons Namensnennung 4.0 International Lizenz veröffentlicht, welche die Nutzung, Vervielfältigung, Bearbeitung, Verbreitung und Wiedergabe in jeglichem Medium und Format erlaubt, sofern Sie den/die ursprünglichen Autor(en) und die Quelle ordnungsgemäß nennen, einen Link zur Creative Commons Lizenz beifügen und angeben, ob Änderungen vorgenommen wurden. 
Die in diesem Artikel enthaltenen Bilder und sonstiges Drittmaterial unterliegen ebenfalls der genannten Creative Commons Lizenz, sofern sich aus der Abbildungslegende nichts anderes ergibt. Sofern das betreffende Material nicht unter der genannten Creative Commons Lizenz steht und die betreffende Handlung nicht nach gesetzlichen Vorschriften erlaubt ist, ist für die oben aufgeführten Weiterverwendungen des Materials die Einwilligung des jeweiligen Rechteinhabers einzuholen.

Weitere Details zur Lizenz entnehmen Sie bitte der Lizenzinformation auf http://creativecommons.org/ licenses/by/4.0/deed.de.

\section{Einhaltung ethischer Richtlinien}

Interessenkonflikt J. Schürmann, M. Mühleck, C. Perler, K. Schmeck und S. Reiter-Theil geben an, dass kein Interessenkonflikt besteht.

Ethische Standards Das Projekt inkl. der Befragungen wurde im Einklang mit nationalem Recht durchgeführt. Das Einverständnis der Teilnehmer*innen wurde eingeholt.

\section{Literatur}

Ackermann S, Balsiger L, Salathé M (2016) Ethikstrukturen an Akutspitälern, Psychiatrischen Kliniken und Rehabilitationskliniken der Schweiz. Bioeth Forum 9:52-59

Aebi M (2019) Die Prävalenz von psychosozialen Belastungen bei jugendlichen Straftätern: eine Metaanalyse. Forens Psychiatr Psychol Kriminol 13:166-177

Albisser Schleger H, Mertz M, Meyer-Zehnder B et al (2019) Klinische Ethik - METAP : Leitlinie für Entscheidungen am Krankenbett. Springer, Berlin Heidelberg

Anderson-Shaw L, Ahrens W, Fetzer M (2007) Ethics consultation in the emergency department. JONAS Healthc Law Ethics Regul 9:32-35

Austin WG, Kirkpatrick H, Flens JR (2011) The emerging forensic role for work product review and case analysis in child access and parenting plan disputes. Fam Court Rev 49:737-749

Baier D (2019a) Jugendkriminalität in der Schweiz. Entwicklungen und Einflussfaktoren. Z Jugendkriminalr Jugendh 3:214-223

Baier D (2019b) Kriminalitätsopfererfahrungen und Kriminalitätswahrnehmungen in der Schweiz : Ergebnisse einer Befragung. Zürcher Hochschule für Angewandte Wissenschaften (ZHAW), Zürich

Barth GM (2003) Ethik in der Gesellschaft - Ethik in der Begutachtung. In: Lehmkuhl U (Hrsg) Ethische Grundlagen in der Kinder und Jugendpsychiatrie und Psychotherapie. Vandenhoeck \& Ruprecht, Göttingen, S 69-88

Beauchamp TL, Childress JF (2013) Principles of biomedical ethics. Oxford University Press, New York

Bessler C, Stiefel D, Barra S et al (2019) Psychische Störungen und kriminelle Rückfälle bei männlichen jugendlichen Gefängnisinsassen. Z Kinder Jugendpsychiatr Psychother 47:73-88

Epstein EG (2012) Preventive ethics in the intensive care unit. AACN Adv Crit Care 23:217-224

Foglia MB, Fox E, Chanko B et al (2012) Preventive ethics: addressing ethics quality gaps on a systems level. Joint Comm J Qual Patient Saf 38:103-111

Heyl J (2008) Early indicators for ethics reviews. Health Care Ethics 16:2-4

Jehle J-M, Hohmann-Fricke S, Albrecht H-J et al (2016) Legalbewährung nach strafrechtlichen Sanktionen. Bundesministerium der Justiz und für Verbraucherschutz, Berlin

Jositsch D, Aebersold P, Schweizer C (2013) Der Fall Carlos: Chronik und Analyse einer Tragödie. Jusletter. https://jusletter.weblaw.ch/juslissues/2013/738/_11877.html. Zugegriffen: 31. Dez. 2020

Kaltiala-Heino R, Eronen M (2015) Ethical issues in child and adolescent forensic psychiatry: a review. J Forensic Psychiatry Psychol 26:759-780

Manzoni P, Baier D, Eberitzsch S (2018) Zum Umgang mit Jugendkriminalität in der Schweiz. In: Dollinger B, Schmidt-Semisch H (Hrsg) Handbuch Jugendkriminalität. Interdisziplinäre Perspektiven. Springer VS, Wiesbaden, S 119-136

Mayring P (2010) Qualitative Inhaltsanalyse : Grundlagen und Techniken. Beltz, Weinheim, Basel

McPherson SB (2012) Major principles in a minor context: Forensic practices involving adolescents. In: Grigorenko EL (Hrsg) Handbook of juvenile forensic psychology and psychiatry. Springer, New York, S 69-81 
Meyer D, Reiter-Theil S (2016) Context-adjusted clinical ethics support (CES) in psychiatry. Accompanying a team through a sensitive period. Clin Ethics 11:70-80

Meyer-Zehnder B, Albisser Schleger H, Tanner S et al (2017) How to introduce medical ethics at the bedside-Factors influencing the implementation of an ethical decision-making model. BMC Med Ethics 18:16

Moher D, Liberati A, Tetzlaff J et al (2009) Preferred reporting items for systematic reviews and metaanalyses: the PRISMA statement. J Clin Epidemiol 62:1006-1012

Morgenstern L (2005) Proactive bioethics screening: a prelude to bioethics consultation. J Clin Ethics 16:151-155

Morrison HL (1986) The forensic evaluation and treatment of children: ethics and values. J Am Acad Psychiatry Law 14:353-359

Niveau G, Welle I (2018) Forensic psychiatry, one subspecialty with two ethics? A systematic review. BMC Med Ethics 19:25

O’Shaughnessy RJ, Andrade HT (2008) Forensic psychiatry and violent adolescents. Brief Treat Crisis Interv 8:27-42

Pavlish C, Brown-Saltzman K, Hersh M et al (2011) Early indicators and risk factors for ethical issues in clinical practice. J Nurs Scholarsh 43:13-21

Pavlish C, Brown-Saltzman K, Fine A et al (2013) Making the call: a proactive ethics framework. HEC Forum 25:269-283

Pavlish C, Brown-Saltzman K, Dirksen KM et al (2015a) Physicians' perspectives on ethically challenging situations: early identification and action. AJOB Empir Bioeth 6:28-40

Pavlish C, Hellyer J, Brown-Saltzman K et al (2015b) Screening situations for risk of ethical conflicts: a pilot study. Am J Crit Care 24:248-256

Quinn KM (1995) Ethical dilemmas in forensic child and adolescent psychiatry. Child Adolesc Psychiatr Clin N Am 4:809-822

Ratner RA (2002) Ethics in child and adolescent forensic psychiatry. Child Adolesc Psychiatr Clin N Am 11:887-904

Reiter-Theil S (2004) Does empirical research make bioethics more relevant? "The embedded researcher" as a methodological approach. Med Health Care Philos 7:17-29

Reiter-Theil S (2016) Initiating and maintaining clinical ethics support in psychiatry. Ten tasks and challenges - and how to meet them. Clin Ethics 11:45-53

Robinson EM, Cage W, Erler K et al (2017) Structure, operation, and experience of clinical ethics consultation 2007-2013: a report from the Massachusetts General Hospital Optimum Care Committee. J Clin Ethics 28:137-152

Sadoff RL, Baird JA (2011) Ethical issues in forensic psychiatry : minimizing harm. John Wiley, Chichester, West Sussex

Scheffold N, Paoli A, Gross J et al (2012) Ethikvisite auf der Intensivstation. Med Klin Intensivmed Notfallmed 107:553-557

Schetky DH (1992) Ethical issues in forensic child and adolescent psychiatry. J Am Acad Child Adolesc Psychiatry 31:403-407

Schürmann J, Reiter-Theil S (2018) Klinische Ethik in der Psychiatrie - aktuelle Entwicklungen, Herausforderungen und Lösungsansätze. In: Sauer T, Schnurrer V, Bockenheimer-Lucius G (Hrsg) Angewandte Ethik im Gesundheitswesen. LIT, Berlin, S 73-90

Sikorski JB, Kuo AD (2004) Forensic psychiatry. In: Wiener JM, Dulcan MK (Hrsg) Textbook of child and adolescent psychiatry. American Psychiatric Publishing, Arlington, S 903-927

Silén M, Ramklint M, Hansson MG et al (2016) Ethics rounds: an appreciated form of ethics support. Nurs Ethics 23:203-213

Soulier MF (2010) Ethics of child and adolescent forensic psychiatry. In: Benedek EP (Hrsg) Child and adolescent forensic mental health. Americal Psychiatric Publishing, Arlington, S 13-27

Souverein F, Dekkers T, Bulanovaite E et al (2019) Overview of European forensic youth care: towards an integrative mission for prevention and intervention strategies for juvenile offenders. Child Adolesc Psychiatry Ment Health 13:6

Strous M (2009) Do dual forensic and therapy roles damage children? J Child Adolesc Ment Health 21:185-186

Syse I, Førde R, Pedersen R (2016) Clinical ethics committees-also for mental health care? The Norwegian experience. Clin Ethics 11:81-86

Thierbach C, Petschick G (2014) Beobachtung. In: Bauer N, Blasius J (Hrsg) Handbuch Methoden der empirischen Sozialforschung. Springer, Wiesbaden, S 855-866 
Unicef (1989) UN-Konvention über die Rechte des Kindes. https://www.unicef.ch/sites/default/files/201808/un-kinderrechtskonvention_de.pdf. Zugegriffen: 31. Dez. 2020

Voskes Y, Weidema F, Widdershoven G (2016) Clinical ethics in forensic psychiatry: Fostering reflection and dialog on the ward through moral case deliberation. Clin Ethics 11:63-69

Wollenburg LM, Claus S, Kieser C et al (2020) Zum Stand der Anwendung klinischer Ethikberatung in deutschen Kliniken für Psychiatrie und Psychotherapie. Psychiatr Prax 47:446-451

Zerby SA, Thomas CR (2006) Legal issues, rights, and ethics for mental health in juvenile justice. Child Adolesc Psychiatr Clin N Am 15:373-390 